\title{
The morphometrical and immunohistochemical investigation of the effect of topiramate on liver and the role of neuropeptide $Y$ receptor in an obese female rat
}

\author{
Yahyazadeh $\mathrm{A}^{1}$, Altunkaynak $\mathrm{BZ}^{2}$, Alkan $\mathrm{I}^{3}$ \\ Department of Histology and Embryology, Faculty of Medicine, Karabuk University, Karabuk, Turkey. \\ yahyazadeh.ahmad@karabuk.edu.tr
}

\begin{abstract}
OBJECTIVE: We aimed to investigate the possible effect of topiramate (TOP, $0.02 \mathrm{mg} / \mathrm{kg} / \mathrm{day}$ ) on the livers in a high-fat diet (HFD)-induced obesity rat model. The other objective was to evaluate the relationship between TOP administration and NPY level using anti-NPY1R antibody.

METHODS: Twenty-four adult female Wistar albino rats were randomly assigned into four equal groups as follow: control (CONT), obese (OBS), TOP, and OBS+TOP. All liver samples were investigated using the stereological analysis, as well as immunohistochemical and histopathological examination.

RESULTS: The total number of hepatocytes was significantly decreased in the OBS+TOP group compared to the CONT group or the OBS group $(p<0.05)$. We found a significant increase in the mean volume of liver in the OBS group compared to the CONT group $(p<0.05)$. Also, the mean volume of liver was significantly decreased in the OBS+TOP group compared to the OBS group $(p<0.05)$.

CONCLUSION: Taken together, our findings suggest that decreased liver volume is possibly attributed to TOP administration via setting the NPY level in the obese rats. Further, the side effects of TOP in combination with health risk of obesity may have led to an increase in hepatotoxicity and the subsequent hepatocyte loss (Fig. 7 , Ref. 56). Text in PDF www.elis.sk KEY WORDS: immunohistochemistry, liver, neuropeptide Y, obesity, rat, topiramate.
\end{abstract}

\section{Introduction}

Topiramate (TOP), a sulfamate-substituted monosaccharide drug, was used for the first time to treat epilepsy and migraine $(1,2)$. Also, it is approved for use as mood stabilizer and in the treatment of post-traumatic stress disorder as well as infantile spasms (3-5). This drug can be quickly absorbed into body, weakly bounded to plasma protein and primarily excreted through kidney, but not extensively metabolized (Kugler and Sachdeo, 1998) (6). In obese subjects, TOP is thought to be beneficial in weight loss due to a reduction in food intake, but it may cause hepatotoxicity $(2,7$, 8). Rosenfeld (9) reported that TOP alone did not cause critical hepatotoxicity compared to other antiepileptic drugs. Andradea et al (10) suggested that drug-induced hepatotoxicity should also be

\footnotetext{
${ }^{1}$ Department of Histology and Embryology, Faculty of Medicine, Karabuk University, Karabuk, Turkey, ${ }^{2}$ Department of Histology and Embryology, Faculty of Medicine, Okan University, Istanbul, Turkey, and ${ }^{3}$ Departments of Histology and Embryology, Okan University, Faculty of Medicine, Istanbul, Turkey
}

Address for correspondence: A. Yahyazadeh, Department of Histology and Embryology, Faculty of Medicine, Karabuk University, Karabuk, Turkey.

Acknowledgement: This work was supported by the Ondokuz Mayis University Project Management Office (TIP.1904.13.024). considered in combination with other risk factors that contribute to the development of liver complications (10).

Obesity, characterized by an excessive fat accumulation, is known as a risk factor for body health. Numerous studies have shown that obesity, one of the 10 most risky diseases, predisposes the living organism to other diseases $(1,11,12)$. Innis (13) documented that obesity caused an increase in circulating inflammatory mediators. In fact, there is a correlation between obesity and damage to liver tissues $(14,15)$.

Neuropeptide Y (NPY) is a 36 amino-acid neuropeptide secreted from neurons in the arcuate nucleus of the hypothalamus. NPY also is a main member of hypothalamic neuropeptide signalling systems that contributes to the regulation of food consumption and energy (16). Expression of NPY may be affected by extrinsic agents. For example, feeding with high-fat diet (HFD) can cause increased expression of hypothalamic NPY and then stimulate the release of NPY. Accordingly, elevated NPY level leads to an increase in food intake and the subsequent weight gain $(17,18)$. NPY can show important physiologic activity in liver tissue via receptors (R) such as NPY1R (19). However, the mechanism that describes signalling pathways regarding the effect of NPY through the activation of NPY1R in liver tissue is still unclear.

Scientific researches with wistar albino rats can provide detailed information and comprehensive results. Also, obesity health problem in humans may be remedied by the results of the animal 
model. There are few studies concerning the effect of TOP on liver tissue in obese subjects. We therefore decided to develop an obesity model with HFD in adult rats and investigate the effect of TOP on liver tissues in HFD-induced obese female rats. We also evaluated the relationship between TOP and NPY level using ant-NPY1R antibody. The present study was a continuation of our previous work in which hypothalamic NPY was significantly increased (20).

\section{Materials and methods}

\section{Animals and experimental procedure}

Ethical approval of our study was granted by the Laboratory Animal Ethics Committee of Ondokuz Mayıs University, Samsun, Turkey (2013/41). Twenty-four 300-350 g adult female Wistar albino rats were used in this study. All subjects were purchased from the Experimental Animal Research and Application Centre of Medicine Faculty of Ondokuz Mayıs University (Samsun, Turkey). During the experiment period, animals were housed in plastic cages under 12:12-h day/night cycle at $22 \pm 2{ }^{\circ} \mathrm{C}$ and $50 \pm 5 \%$ humidity. Animals had ad libitum access to food and tap water. The OBS and OBS+TOP groups were fed with HFD containing $40 \%$ fat (21). Rats were selected and divided randomly into four equal groups $(n=6)$ as follow:

1. Control (CONT) group: Rats were not treated with any substance. All animals were fed with commercial rat diet (7-10\% fat, $68-70 \% \mathrm{CH}, 18-20 \%$ protein, $1-2$ vitamins and minerals; $210 \mathrm{kcal} / 100 \mathrm{~g} /$ day) during the fifteen-week experimental pe$\operatorname{riod}(14)$.

2. Obese (OBS) group: Rats were fed with HFD (40\% fat, $40-42 \% \mathrm{CH}, 18-20 \%$ protein $1-2 \%$ vitamins and minerals; $210 \mathrm{kcal} / 100 \mathrm{~g} /$ /day) during the nine-week experimental pe$\operatorname{riod}(14)$.

3. Topiramate (TOP) group: Rats were fed with commercial rat diet and treated intraperitoneally (i.p.) with TOP $(0.06 \mathrm{mg} / \mathrm{kg} /$ day; Powder, Sigma-Aldrich, St Louis, MO, USA) during the six-week experimental period.

4. Obese + topiramate (OBS+TOP) group: Rats were fed with HFD during the nine-week experimental period and treated intraperitoneally with TOP $(0.02 \mathrm{mg} / \mathrm{kg} /$ day $)$ during the six-week experimental period.

Our experimental procedure was carried out during the 15-week experimental period. HFD-fed rats were considered as obese rats at the end of the 9th week. Subsequently, both the OBS+TOP and TOP groups were treated i.p. with $0.06 \mathrm{mg} / \mathrm{kg}$ topiramate from the 10th to the 15 th week.

At the end of the 15th week, all animals were anesthetized with both ketamine $(100 \mathrm{mg} / \mathrm{kg}$ body weight; Sigma Chemical Comp., St. Louis, MO, USA) and prilocaine hydrochloride (2.5 $\mathrm{mg} / \mathrm{kg}$ body weight; Sigma Chemical Comp., St.
Louis, MO, USA). The liver tissues were then immediately dissected for the stereological, histopathological and histopathological studies.

\section{Histological studies}

All liver samples were fixed in $10 \%$ formalin solution, then dehydrated through graded series of alcohols, cleared in xylene and impregnated with paraffin. Liver samples were cut at $8-\mu \mathrm{m}$ thickness and 1/280 interval using a rotary microtome (Leica RM 2135, Leica Instruments, Nussloch, Germany) with disposable metal blades. Consecutive sections were obtained from each sample based on the systematic random sampling technique. All sections were mounted on glass slides and then stained with haematoxylin-eosin for histopathological examination and stereological analysis (22).

\section{Stereological analyses}

The Physical fractionator and Cavalieri methods were used to estimate the hepatocyte number and mean volumes of sinusoids and livers (Fig. 1) $(23,24)$.

The mean volume of sinusoids and livers was calculated using both the Cavalieri and the point-counting methods $(25,26)$. The systematic randomly sampling technique was performed to obtain the accurate and valuable results. According to the pilot study, we determined the point density of the point-counting grid to specify whether the grid size is appropriate. Images of each section were photographed using a light microscope (Leica RM 2135, Leica Instruments, Nussloch, Germany). The counting frame grid was then randomly superimposed on images, and the number of points
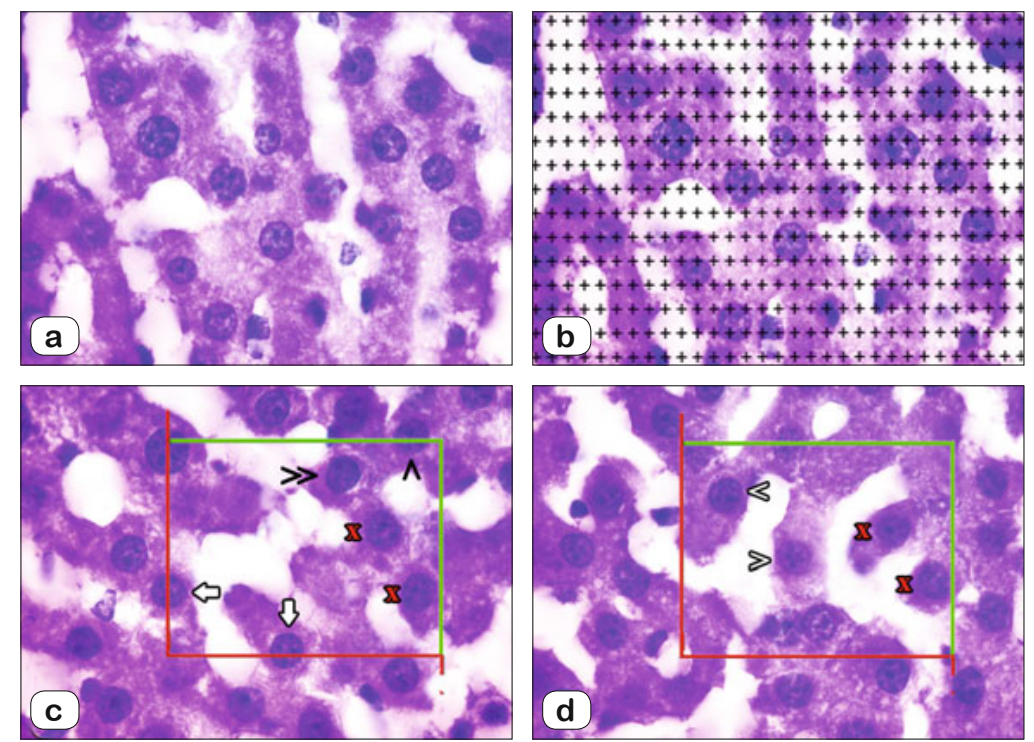

Fig. 1. Stereological procedures of the Cavalieri (a and b) and physical disector (c and d) methods. (c), reference section; (d), look-up section; X, hepatocytes detected in both the reference and look-up sections; black arrowhead, hepatocytes hit the inclusion line of frame in the reference section; double arrowheads, hepatocytes detected in the reference section; white arrowheads, hepatocytes detected in the look-up section; white arrow, hepatocytes hit the exclusion line of frame in the reference section. Hepatocytes detected in the reference section but not in the look-up section are counted. 
656-662

hitting on each field was counted. Finally, the mean volumes of the regions of interest were calculated as $(27,28)$ :

$\mathrm{V}($ total volume $)=\mathrm{t} \times \sum \mathrm{A}$

Where, " $t$ " is the total thickness of each section and interval, and $\Sigma \mathrm{A}$ is the total area of the regions of interest in all sections. Also, the term $\Sigma \mathrm{A}$ is represented in this equation:

$$
\sum \mathrm{A}=\mathrm{a}(\mathrm{p}) \times \sum \mathrm{P}
$$

Where, $\mathrm{a}(\mathrm{p})$ is the interval point area, and $\Sigma \mathrm{P}$ is the total number of points hitting on the regions of interest. Thereby, the above formula can be displayed as:

$$
\mathrm{V}(\text { total volume })=\mathrm{t} \times \mathrm{a}(\mathrm{p}) \times \sum \mathrm{P}
$$

The total number of hepatocytes was calculated by means of the physical fractionator method. A pilot study was performed to determine the sampling and counting strategy. In the present study, three steps were summarized to apply the rules of physical fractionator using the systematic randomly sampling method. The first step was the piece sampling fraction (PSF) that produces the piece similar in size. The second was the section sampling fraction (SSF) providing the disector pairs. The third was the area sampling fraction (ASF). All sections were photographed by a light microscope (Leica RM 2135, Leica Instruments, Nussloch, Germany) at a magnification of $40 \mathrm{x}$. To count particle profiles, the counting fields were randomly sampled in both the reference and look-up sections. These counting fields must be carefully orientated to each other. Then, unbiased counting frames were superimposed on the same counting field. Particles were estimated according to the physical dissector principle using the ImageJ program. Particles observed in the reference section but not in the look-up section were accepted for counting (27). The hepatocyte number was calculated as follow:

$$
\mathrm{TN}(\text { total number })=\sum \mathrm{Q}^{-} \frac{1}{\mathrm{PSF}} \times \frac{1}{\mathrm{SSF}} \times \frac{1}{\mathrm{ASF}}
$$

Where, $\mathrm{TN}$ is the total number of hepatocytes, $\Sigma \mathrm{Q}^{-}$is the number of counted particles.

The number of hepatocytes counted in each animal was validated by the coefficient of error (28). Also, the coefficient of variation $(\mathrm{CV})$ identified whether animals in each group were adequate (29). On the other hand, sample size in each group was determined using CV value. CV value less than 0.2 showed that results from this animal number were valid.

\section{Immunohistochemical procedure}

Anti-NPY1R antibody (ab216966) were conducted on 8- $\mu \mathrm{m}$ thick liver sections. Briefly, sections were incubated with $3 \% \mathrm{H}_{2} \mathrm{O}_{2}$ at $37{ }^{\circ} \mathrm{C}$ to block the endogenous peroxidases for $15 \mathrm{~min}$. Sections were treated with ficin solution, followed by SuperBlock blocking buffer to eliminate non-specific immunoreactivity for $7 \mathrm{~min}$. Subsequently, sections were applied to anti-NPY1R antibody at $37^{\circ} \mathrm{C}$ in humid medium for $120 \mathrm{~min}$, then washed in PBS buffer three times for $5 \mathrm{~min}$. These sections were then were treated with biotinylated secondary antibody for $30 \mathrm{~min}$. Immunological acti-

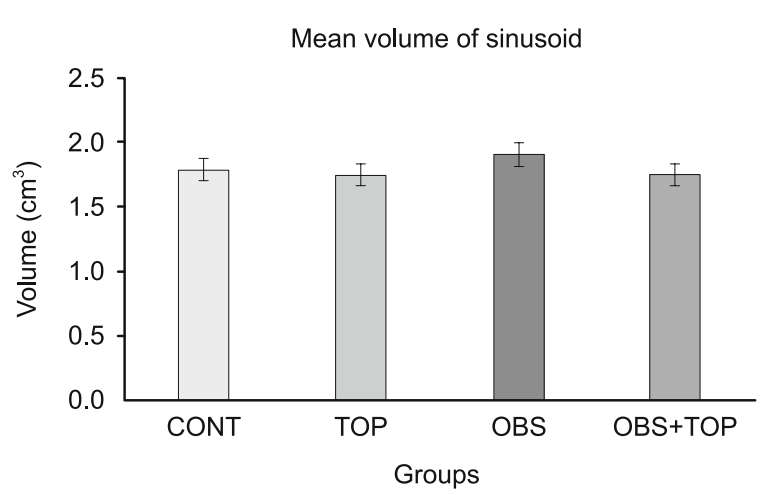

Fig. 2. The mean volume of sinusoids in the CONT, TOP, OBS, OBS+TOP groups. CONT, control group; TOP, topiramate group; OBS, obese group; OBS+TOP, obese + topiramate group.

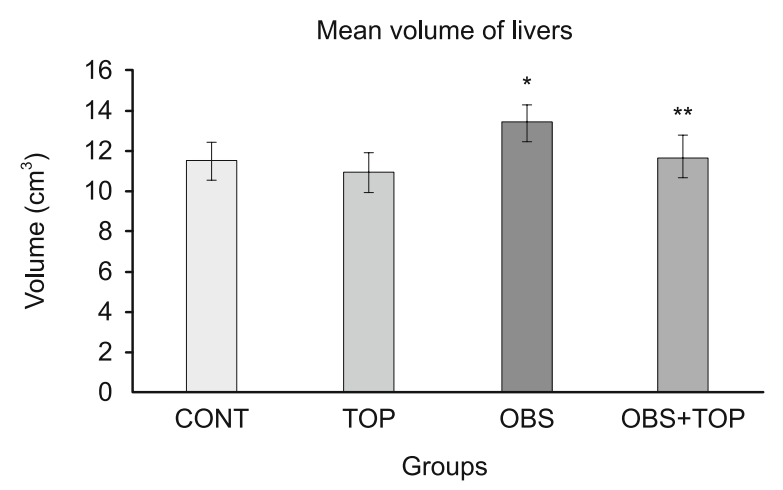

Fig. 3. The mean volume of livers in the CONT, TOP, OBS, OBS+TOP groups. *, shows a significant difference from the CONT and TOP groups; **, shows a significant difference from the OBS group. CONT, control group; TOP, topiramate group; OBS, obese group; OBS+TOP, obese+topiramate group.

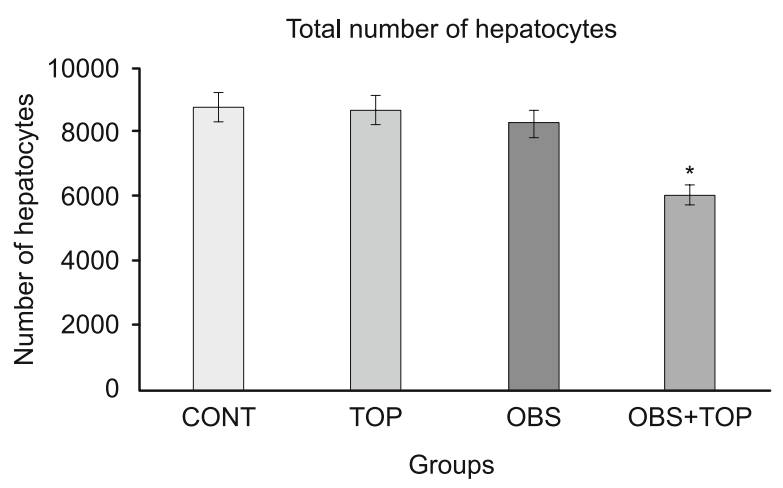

Fig. 4. The total number of hepatocytes $\left(\times 10^{4}\right)$ in the CONT, TOP, OBS, OBS+TOP groups. *, shows a significant difference from the CONT, TOP and OBS groups. CONT, control group; TOP, topiramate group; OBS, obese group; OBS+TOP, obese + topiramate group.

vity was assessed with a streptavidin-biotin complex kit (ScyTek Laboratories, SitoGen Biomedikal Ltd; Istanbul, Turkey). Finally, sections were counterstained with Mayer's haematoxylin. Immunohistochemically stained liver samples were analysed using a light microscope (Leica, LDM 4000, Wetzlar, Germany). 


\section{Statistical analyses}

The data were statistically analysed using IBM version 25.0 SPSS software (SPSS Inc., Chicago, IL, USA). We used One-way ANOVA and the Tukey's post hoc test. P value less than 0.05 was considered statistically significant, and results were expressed as mean \pm SEM.

\section{Results}

\section{Stereological results}

The total number of hepatocytes and the mean volume of liver and sinusoids were calculated by stereological methods. Stereological results are given in Figures 2, 3 and 4. All animals in each group were included in each analysis $(6 / 6)$.

\section{Mean volumes of sinusoids and livers}

We found that there was no significant difference in the mean volume of sinusoids between the CONT group and experimental groups.

The mean volume of liver was significantly increased in the OBS group compared to the CONT group $(\mathrm{p}<0.03)$ or the TOP groups $(\mathrm{p}<0.00)$. There was a significant decrease in the OBS+TOP group compared to the OBS group $(\mathrm{p}<0.04)$ (Figs 1 and 2).

\section{Total number of hepatocytes}

While there was no significant difference between the OBS and CONT groups, the total number of hepatocytes was significantly decreased in the OBS+TOP group compared to the CONT ( $p$ $<0.02)$ and TOP $(\mathrm{p}<0.03)$ groups. We have also found no significant difference between the TOP group and the OBS group or the CONT groups (Fig. 4).

\section{Immunohistochemical results}

Positive immunohistochemical staining with Anti-NPY1R antibody was observed as dark brown in the liver of all groups (Fig. 5). Positive-NPY1R immunohistochemical staining was slight in the CONT group. By contrast, strong positive staining was detected in the OBS group. Also, immunohistochemical findings exhibited the slight staining in the TOP group and even less than CONT group. In the OBS+TOP group, positive immunostaining was lower than in those of the OBS group.

\section{Histopathological results}

Histological and histopathological findings are listed in Figures 6 and 7. We observed that hepatic tissue's architectures appeared normal in the
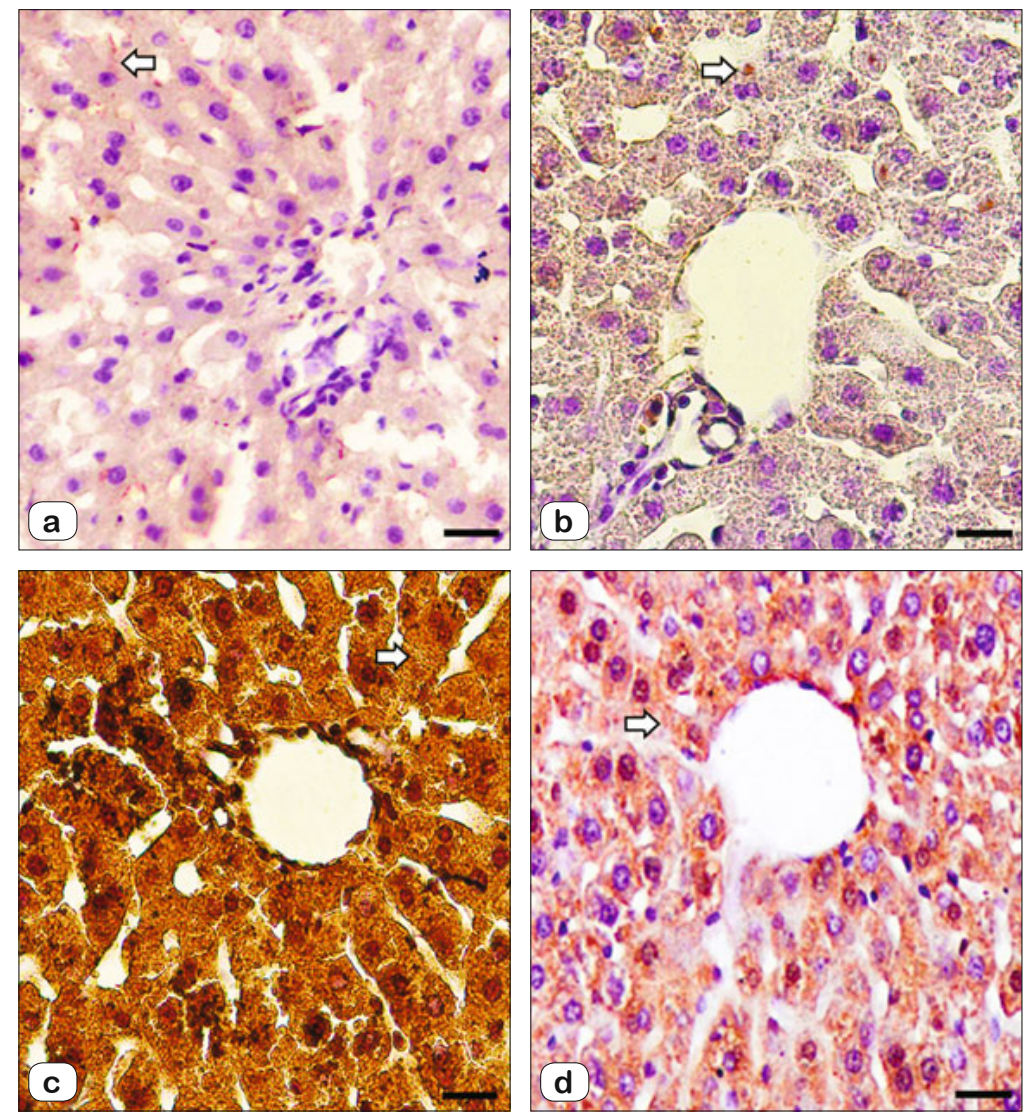

Fig. 5. Immunohistochemical micrographs of liver tissues stained with Anti-NPY in the CONT (a), TOP (b), OBS (c) and OBS+TOP (d) groups. Arrow, positively stained cell. CONT, control group; TOP, topiramate group; OBS, obese group; OBS+TOP, obese + topiramate. Scale bars $=25 \mu \mathrm{m}$.
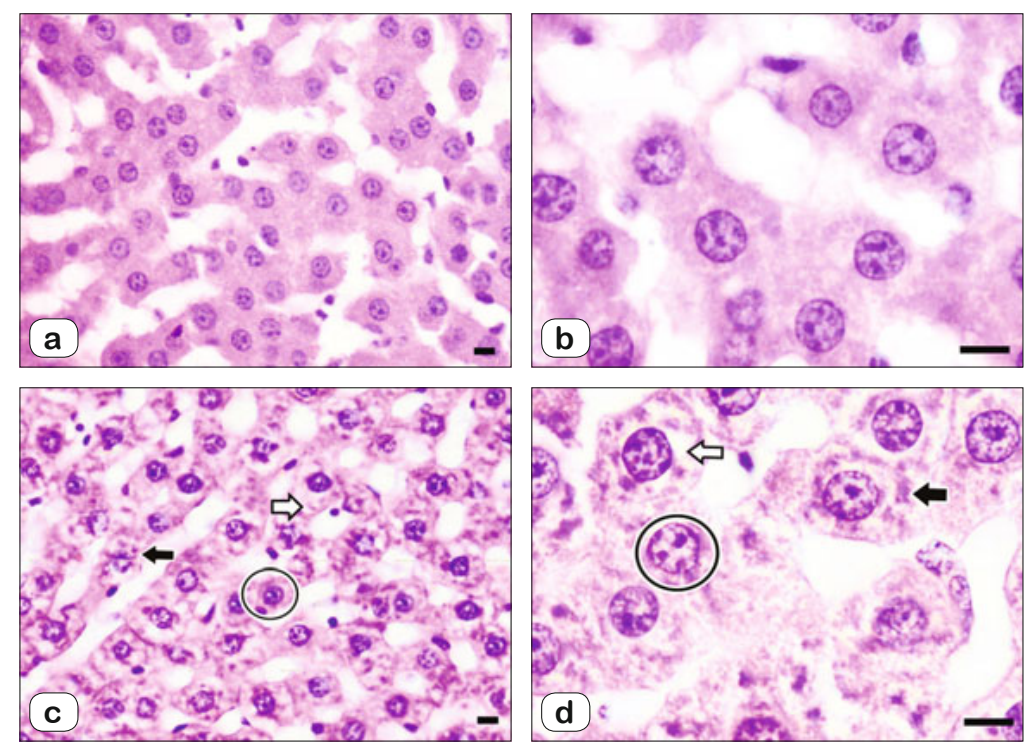

Fig. 6. Light micrographs of liver tissues in the CONT (a and b) and TOP groups (c and d). White arrow, vacuoles; black arrow, scattered eosinophilic spots; circle, basophilic staining of hepatocyte nuclei. CONT, control group; TOP, topiramate group. Scale bars $=12.5 \mu \mathrm{m}$. 

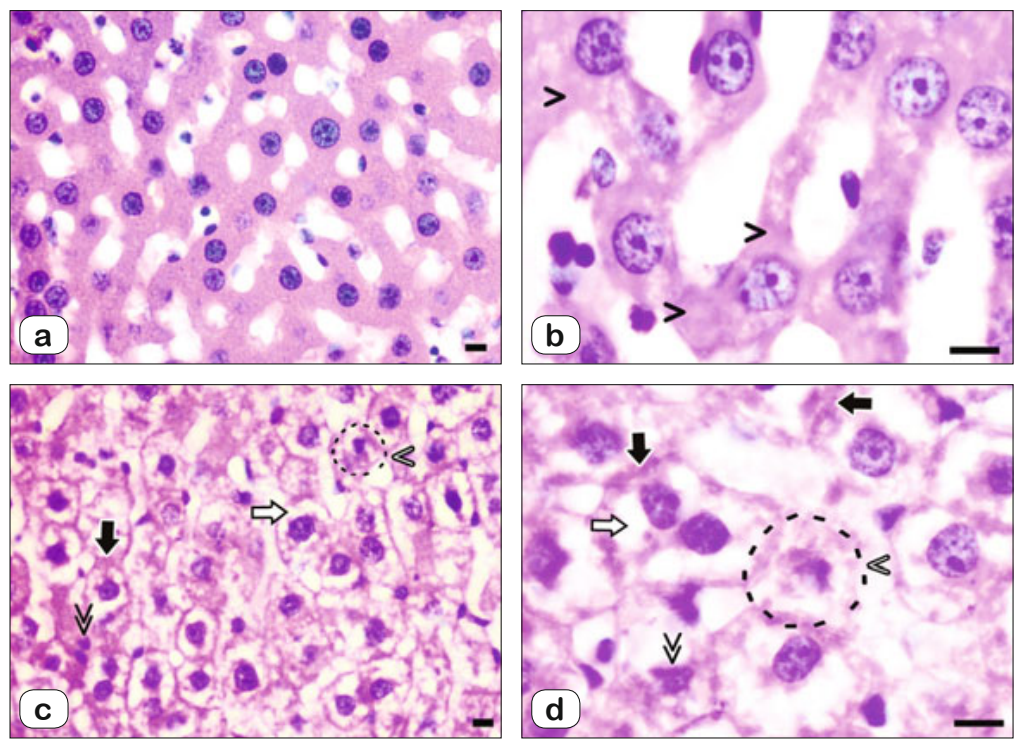

Fig. 7. Light micrographs of liver tissues in the OBS (a and b) and OBS+TOP (c and d) groups. Black arrowhead, ambiguously stained cytoplasm of hepatocyte; white arrow, vacuoles; black arrow, scattered eosinophilic spots; white arrowhead, degenerated hepatocytes; double black arrow, pyknosis. OBS, obese group; OBS+TOP, obese + topiramate group. Scale bars $=\mathbf{1 2 . 5} \mu \mathrm{m}$.

CONT group (Fig. 6a and b). In the TOP group, hepatocytes were stained relatively less than those of the CONT group; however, no degenerated hepatocytes were found (Fig. $6 \mathrm{c}$ and d). Also, we found less eosinophilic cytoplasm, dispersed vacuoles, scattered eosinophilic spots, basophilic staining of nuclei in hepatocytes. In the OBS group, cytoplasm of hepatocytes was stained ambiguously (Fig. 7a and b). We also found marked changes in hepatocytes of the OBS+TOP group such as degeneration, less eosinophilic cytoplasm, extensive vacuoles and variable size of nuclei (Fig. 7c and d). In addition, pyknotic nuclei with irregular borders were detected as well.

\section{Discussion}

TOP and obesity are two main factors that may affect liver tissue. TOP as antiepileptic drug has been shown to reduce the body weight in obese subjects. Obesity also can adversely impact the liver that contributes to many metabolic processes (30). Our previous study showed that feeding with HFD caused an increase in NPY levels in the rat hypothalamus, leading to obesity (20). There is few information regarding the interaction between obesity and TOP administration in liver tissue. We therefore decided to investigate the possible effect of TOP on the liver in an obese rat model. The hepatocytes number, as well as mean volumes of sinusoids and livers were investigated by means of stereological methods $(22,23)$.

Today, public health is threatened with obesity, especially in developed countries due to imbalance of energy (31). The velocity rate of dietary intake normally depends on the amount of both fat and carbohydrate present in human deposits. Feeding with HFD can cause fatty liver, which may result in advanced non-alcoholic steatohepatitis and cirrhosis $(32,33)$. Fatty liver with environmental and genetic factors causes an increase oxidative stress in hepatic tissue (34). Obesity can be caused by various agents such as psychogenic obesity, neurogenic disorders, genetics and the environment (35-37). Moreover, obesity may be due to altering the regulation of food intake at the centre of hypothalamic hunger and satiety (38). NPY as a neuropeptide plays an important role in regulation of food consumption, which is mostly found in brain (39). Some studies reported that obesity caused metabolic disorder and inflammation, as well as steatosis in the liver $(40,41)$.

Our volumetric results that the mean volume of liver was significantly increased in the OBS group compared to the CONT group is consistent with earlier reports. Park et al (38) reported that NPY expression was significantly increased in hypothalamus neurons in HFD-induced obese rats. Therefore, fat accumulation in the liver can be attributed to an increase in food intake under the influence of NPY (20). We also found that the mean volume of liver in the OBS+TOP group was significantly decreased compared to the OBS group. This reduction may have resulted from decreased NPY level following TOP administration, which causes a decrease in food intake (17). Kazerooni and Lim (42) reported that TOP could cause weight loss and changes in body mass index in overweight and obese patient.

We also found no significant difference in the total number of hepatocytes in the TOP group compared to the CONT group or the OBS group, which suggests no adverse effect for TOP. To the contrary, the decreased number of hepatocytes in the OBS+TOP group compared to the CONT group or the OBS groups may have been attributed to toxic activity of TOP in association with obesity.

TOP is not only involved in lipid metabolism by modulating hepatic gene expression but also has a harmful effect on liver tissue $(43,44)$. Reports regarding the effect of TOP on biosystem have been inconclusive. Some studies have documented the ameliorative effect of TOP on body organs (45-47). To the contrary, Khivsara et al (48) reported that TOP may induce toxicity in hepatic tissue.

Hypothesis that TOP induces hepatoxicity may be due to its influence on cell mitochondria (49). TOP that interferes with mitochondrial function may result in mitochondrial disorder (50). Moreover, TOP as a causal complex factor for the liver can increase the ammonia, which enhances the rate of damage $(51,52)$. Dogson et al (53) reported that inhibition of TOP-induced type $\mathrm{V}$ mitochondrial carbonic anhydrase may lead to hyperammonemia (53). The reduction of bicarbonate synthesis can decrease detoxification of ammonia that causes damage to hepatic tissue (54). Another study also documented the adverse effect of TOP on hepatic molecules in obese rat liver (55). Mechanism that describes the decreased hepatocyte number caused by TOP has not been reported in previous studies. 
Our histological results exhibited that the architectures of hepatic tissue appeared normal in the CONT group. We observed some changes in the hepatic tissue in the TOP group; however, no hepatocyte degeneration was detected. Also, hepatocyte cytoplasm of OBS group was stained ambiguously. Likewise, remarkable structural damage to hepatocytes was detected in the OBS+TOP group, but this was more than in the OBS group or TOP group. This was possibly due to destructive effect of TOP on the liver in obese model rats. It has been documented that prolonged administration of TOP causes a significant decrease in antioxidant capacity and a subsequent hepatotoxicity in young obese rats $(43,56)$.

We observed strongly positive staining with anti-NPY1R antibody in the OBS group compared to the CONT group. Administration of TOP caused a decrease in positive anti-NPY1R staining in the OBS+TOP than in the OBS group. In fact, there is a relationship between administration of TOP and decrease in NPY level.

The results of animal experiments have been able to save many human lives, and these studies have played a major role in discovering the underlying mechanism of chemical molecules used in the body. Despite the similarities between the basic chemical properties of the species, further studies should be carried out to avoid further damage to human due to the inaccurate results of these experiments.

\section{Conclusion}

One of the main causes of obesity is feeding with HFD, which is growing significantly worldwide. HFD-induced obesity significantly increased the mean volume of liver. Also, administration of TOP in combination with obesity caused a significant decrease in the mean volume of liver and the hepatocyte number. Decreased liver volume may have resulted from decrease in the NPY level. We suggest that topiramate causes liver complications only in HFDinduced obese rats. Much effort is needed to find out unknown information concerning the possible effect of TOP on liver in obese rat model. We also suggest that other substances with lower side effects should be examined to improve obesity.

\section{References}

1. Altunkaynak BZ, Altunkaynak ME. Relationship of body weight and volume of liver. A morphometrical and stereological study. Saudi Med J 2007; 28 (6): 891-895.

2. Richard D, Ferland J, Lalonde J, Samson P, Deshaies Y. Influences of Topiramate in the regulation of energy balance. Nutrition 2000; 16 (10): 961-966.

3. Andrus MR, Gilbert E. Treatment of civilian and combat-related posttraumatic stress disorder with topiramate. Ann Pharmacother 2010; 44 (11): $1810-1816$.

4. Letmaier M, Schreinzer D, Wolf R, Kasper S. Topiramate as a mood stabilizer. Int Clin Psychopharmacol 2001; 16 (5): 295-298.

5. Glauser TA, Clark PO, Strawsburg R. A pilot study of topiramate in the treatment of infantile spasms. Epilepsia1998; 39 (12): 1324-1328.

6. Kugler SL, Sachdeo RC. Topiramate efficacy in infancy Pediatr. Neurol1998; 19: 320-322.
7. Barnes D, Yaffe K. The projected effect of risk factor reduction on Alzheimer's disease prevalence. Lancet. Neurol 2011; 10 (9): 819-828.

8. Tremblay A, Chaput JP, Bérubé-Parent S, Prud'homme D, Leblanc C, Alméras $\mathbf{N}$ et al. The effect of topiramate on energy balance in obese men: a 6-month double-blind randomized placebocontrolled study with a 6-month open-label extension. Eur J Clin Pharmacol 2007; 63 (2): 123-134.

9. Rosenfeld WE. Topiramate: A review of preclinical, pharmacokinetic, and clinical data. Clin Ther 1997; 19 (6): 1294-1308.

10. Andradea RJ, Camargo R, Lucena MI, González-Grande R. Causality assessment in drug-induced hepatotoxicity. Expert Opin Drug Saf 2004; 3 (4): 329-344.

11. Kim JK, Song YR, Kwon YJ, Kim HJ, Kim SG, Ju YS. Increased body fat rather than body weight has harmful effects on 4-year changes of renal function.n in the general elderly population with a normal or mildly impaired renal function. Clin Interv Aging 2014; 9: 1277-1286.

12. Musaad S, Haynes EN. Biomarkers of obesity and subsequent cardiovascular events. Epidemiol Rev 2007; 29: 98-114.

13. Innis SM. Dietary lipids in early development: relevance to obesity, immune and inflammatory disorders. Curr Opin Endocrinol Diabetes Obes 2007; 14 (5): 359-364.

14. Altunkaynak BZ. Effects of high fat diet induced obesity on female rat livers (a histochemical study). Eur J Intern Med 2005; 2: 100-109.

15. Masarone M, Federico A, Abenavoli L, Loguercio C, Persico M. Non alcoholic fatty liver: epidemiology and natural history. Rev Recent Clin Trials 2014; 9 (3): 126-133.

16. Besnier E, Clavier T, Tonon MC, Pelletier G, Dureuil B, Castel H et al. Anesthetic drugs modulate feeding behavior and hypothalamic expression of the POMC polypeptide precursor and the NPY neuropeptide. BMC Anesthesiol 2018; 18 (1): 96.

17. Verrotti A, Scaparrotta A, Agostinelli S, Di Pillo S, Chiarelli F, Grosso S. Topiramate-induced weight loss: a review. Epilepsy Res 2011; 95 (3): 189-199.

18. Nguyen LT, Saad S, Tan Y, Pollock C, Chen H. Maternal high-fat diet induces metabolic stress response disorders in offspring hypothalamus. J Mol Endocrinol 2017; 59 (1): 81-92.

19. Dai W, Liu Y, Zhang Y, Sun Y, Sun C, Zhang Y et al. Expression of neuropeptide $\mathrm{Y}$ is increased in an activated human HSC cell line. Sci Rep 2019; 9 (1): 9500.

20. Alkan I, Altunkaynak BZ, Altun G, Erener E. The Investigation of the Effects of Topiramate on the Hypothalamic Levels of Fat Mass/ Obesity-Associated Protein and Neuropeptide Y in Obese Female Rats. Nutr Neurosci 2019; 22 (4): 243-252.

21. Sayan H, Ozacmak VH, Ozen OA, Coskun O, Arslan SO, Sezen SC et al. Beneficial effects of melatonin on reperfusion injury in rat sciatic nerve. J Pineal Res 2004; 37 (3): 143-148.

22. Yahyazadeh A, Altunkaynak BZ. Protective effects of luteolin on rat testis following exposure to $900 \mathrm{MHz}$ electromagnetic field. Biotech Histochem 2019; 94 (4): 298-307.

23. Ulubay M, Yahyazadeh A, Deniz ÖG, Kıvrak EG, Altunkaynak BZ, Erdem G et al. Effects of prenatal $900 \mathrm{MHz}$ electromagnetic field exposures on the histology of rat kidney. Int J Radiat Biol 2015; 91 (1): 35-41.

24. Altunkaynak BZ, Akgül N, Yahyazedeh A, Makaracı E, Akgül HM. A stereological study of the effects of mercury inhalation on the cerebellum. Biotech Histochem 2019; 94 (1): 42-47. 
656-662

25. Altunkaynak ME, Altunkaynak ME, Akgül N, Yahyazadeh A, Altunkaynak BZ, Türkmen AP et al. A stereological and histopathological study of the effects of exposure of male rat testes to mercury vapor. Biotech Histochem 2015; 90 (7): 529-534.

26. Yahyazedeh A, Altunkaynak BZ, Akgül N, Akgül HM. A histopathological and stereological study of liver damage in female rats caused by mercury vapor. Biotech Histochem 2017; 92 (5): 338-346.

27. Kaplan S, Gökyar A, Unal B, Tunç AT, Bahadir A, Aslan H. A simple technique for localizing consecutive fields for disector pairs in light microscopy: application to neuron counting in rabbit spinal cord following spinal cord injury. J Neurosci Methods 2005; 145 (1-2): 277-284.

28. Tunç AT, Turgut M, Aslan H, Sahin B, Yurtseven M, Kaplan S. Neonatal pinealectomy induces Purkinje cell loss in the cerebellum of the chick: a stereological study. Brain Res 2006; 1067 (1): 95-102.

29. Yahyazadeh A, Altunkaynak BZ. Investigation of the neuroprotective effects of thymoquinone on rat spinal cord exposed to $900 \mathrm{MHz}$ electromagnetic field. J Chem Neuroanat 2019; 100: 101657.

30. Aleksandrova K, Stelmach-Mardas M, Schlesinger S. Obesity and Liver Cancer. Recent Results Cancer Res 2016; 208: 177-198.

31. Kramer H, Luke A. Obesity and kidney disease: a big dilemma. Curr Opin Nephrol Hypertens 2007; 16 (3): 237-241.

32. Angulo P. Nonalcoholic fatty liver disease. N Engl J Med 2002; 346 (16): 1221-1231.

33. Ji G, Zhao X, Leng L, Liu P, Jiang Z. Comparison of dietary control and atorvastatin on high fat diet induced hepatic steatosis and hyper lipidemia in rats. Lipid Health Dis 2011; 10: 23-33.

34. Day CP, James OF. Steatohepatitis: a tale of two hits. Gastroenterology 1998; 114 (4): 842-845.

35. Altunkaynak BZ, Ozbek E, Muhammed E Altunkaynak ME. A Stereological and histological Analysis of Spleen on Obese Female Rats, Fed with High Fat Diet. Saudi Med J 2007; 28 (3): 353-357.

36. Sengier A. Multifactorial etiology of obesity: nutritional and central aspects. Rev Med Brux 2005; 26 (4): 211-214.

37. Wangensteen T, Undlien D, Tonstad S, Retterstøl L. Genetic causes of obesity. Tidsskr Nor Laegeforen 2005; 125 (22): 3090-3093.

38. Park HJ, Kim JH, Shim I. Anti-obesity Effects of Ginsenosides in High-Fat Diet-Fed Rats. Chin. J Integr Med 2019; 25 (12): 895-901.

39. Yue Z, Zhao Z. Feeding regulation by neuropeptide $Y$ on Asian corn borer Ostrinia furnacalis. Arch Insect Biochem Physiol 2017; 95 (2).

40. Lee JS, Jun DW, Kim EK, Jeon HJ, Nam HH, Saeed WK. Histologic and Metabolic Derangement in High-Fat, High-Fructose, and Combination Diet Animal Models. Sci World J 2015; 2015: 306326.

41. Shimada Y, Kuninaga S, Ariyoshi M, Zhang B, Shiina Y, Takahashi Y et al. E2F8 promotes hepatic steatosis through FABP3 expression in dietinduced obesity in zebrafish. Nutr Metab (Lond) 2015; 12: 17.
42. Kazerooni R, Lim J. Topiramate-Associated Weight Loss in a Veteran Population. Mil Med 2016; 181 (3): 283-286.

43. Huang J, Ren RN, Chen XM, Ye LY. An experimental study on hepatotoxicity of topiramate in young rats. Zhongguo Dang Dai Er Ke Za Zhi 2007; 9 (1): 54-58.

44. Liang Y, She P, Wang X, Demarest K. The messenger RNA profiles in liver, hypothalamus, white adipose tissue, and skeletal muscle of female Zucker diabetic fatty rats after topiramate treatment. Metabolism 2006; 55 (10): 1411-1419.

45. Park HJ, Kim HJ, Park HJ, Ra J, Zheng LT, Yim SV et al. Protective effect of topiramate on kainic acid-induced cell death in mice hippocampus. Epilepsia 2008; 49 (1): 163-167.

46. Cure E, Cure MC, Tumkaya L, Kalkan Y, Aydin I, Kirbas A et al. Topiramate ameliorates abdominal aorta cross-clamping induced liver injury in rats. Saudi J Gastroenterol 2014; 20 (5): 297-303.

47. Cure MC, Cure E, Tumkaya L, Kalkan Y, Aydin I, Kirbas A et al. Topiramate has protective effect on renal injury. Bratisl Lek Listy 2015; 116 (4): 259-263.

48. Khivsara A, Raj JP, Hegde D, Rao M. Topiramate-induced acute liver injury: A rare adverse effect. Indian J Pharmacol 2017; 49 (3): 254-256.

49. Kudin AP, Debska-Vielhaber G, Vielhaber S, Elger CE, Kunz WS. The mechanism of neuroprotection by topiramate in an animal model of epilepsy. Epilepsia 2004; 45 (12): 1478-1487.

50. Finsterer J, Zarrouk Mahjoub S. Mitochondrial toxicity of antiepileptic drugs and their tolerability in mitochondrial disorders. Expert Opin Drug Metab Toxicol 201; 8 (1): 71-79.

51. Fontana RJ, Watkins PB, Bonkovsky HL, Chalasani N, Davern T, Serrano J et al. Drug-induced Liver Injury Network (DILIN) prospective study: rationale, design, and conduct. Drug Saf 2009; 32 (1): 55-68.

52. Tsien MZ, Cordova J, Qadir A, Zhao L, Hart J, Azzam R. Topiramate-induced Acute Liver Failure in a Pediatric Patient: a Case Report and Review of Literature. J Pediatr Gastroenterol Nutr 2016; 63 (3): e37-38.

53. Dogson SJ, Shank RP, Maryanoff BE. Topiramate as an inhibitor of carbonic anhydrase isoenzymes. Epilepsia 2000; 41 Suppl 1: S35-S39.

54. Latour P, Biraben A, Polard E, Bentué-Ferrer D, Beauplet A, Tribut O et al. Drug induced encephalopathy in six epileptic patients: topiramate? valproate? or both? Hum Psychopharmacol 2004; 19 (3): 193-203.

55. El-Abhar HS, Schaalan MF. Topiramate-induced modulation of hepatic molecular mechanisms: an aspect for its anti-insulin resistant effect. PLoS One 2012; 7 (5): e37757.

56. Wang Y, Li JY, Han M, Wang WL, Li YZ. Prevention and treatment effect of total flavonoids in Stellera chamaejasme L. on nonalcoholic fatty liver in rats. Lipids Health Dis 2015; 14: 85.

Received April 7, 2020. Accepted May 20, 2020. 